Luciana MIRRI, Bologna

\title{
RAPPORTO TRA CONTINENZA E MATRIMONIO IN S. GIROLAMO
}

La tradizione ascetico-mistica inaugurata dal grande pensiero patristico non intende negare alle nozze la loro bontà intrinseca in linea di principio, ma nell'esaltazione della continenza per il Regno dei cieli tesse piuttosto le lodi dei nuovi ideali cristiani che di per sé promuovono sia i valori del matrimonio, sia la dignità della donna. Girolamo, grande apologeta della vita consacrata nel celibato per Cristo, si colloca in tale quadro culturale. Per questo, diversi suoi contenuti sull'ascesi monastica e sulla realtà delle nozze vengono maggiormente focalizzati dal confronto del pensiero da lui espresso su entrambe le realtà.

La problematica non concerne soltanto verginità e matrimonio, ma pure lo stato di vedovanza e la dibattuta questione delle seconde nozze. Anzi, proprio quest'ultima pare essere stata assai più al centro del dibattito del Cristianesimo dei primi secoli che non la prima. L'asceta dalmata stesso vi dedica non minor attenzione che all'esaltazione della stessa verginità, rivelando in tal modo, nella Grande Chiesa del IV secolo, ancora viva l'incertezza di un pensiero teologico non del tutto chiaro riguardo al matrimonio in sé. Ne sono prova anche i vari movimenti ereticali che lo intaccano e che Girolamo dovette confutare a salvaguardia della fede ecclesiale ${ }^{1}$. Oltre agli encratiti, sul pensiero dominante esercitarono forte, influsso la distinzione platonica tra voṽ gnosticismo, che ne esasperò la teoria, lo stoicismo e, più ancora, la dottrina di Origene sulla „doppia creazione" 2 .

${ }^{1}$ Cf. Ad Ephesios (= Ad Eph) III 5, 25, PL 26, 566: „, haereticis [...] nuptias repudiandas putant ... Quibus brevius respondendum, passiones hic et immunditiam, et luxuriam ab Apostolo inter maritum et uxorem vetari, non sanctam conjunctionem: alioquin si conjugium omnino prohibebat, quid necesse fuit dicere, «uxores vestras» ... «Vestras» enim proprie matrimonium sonat".

${ }^{2}$ Cf. T. Špidlík - I. Gargano, La spiritualità dei Padri greci e orientali, Roma 1983, 69-85; T. Špidlík, La spiritualità dell'Oriente cristiano, Roma 1985, 55-61, 79-83, 99-104, 231-234; E. Testa, Il Peccato di Adamo nella Patristica (Gen III), Gerusalemme 1970, 172; La coppia nei Padri, Milano 1991, 17-41; C. Mohrmann (a cura di), Vita di Martino. Vita di Ilarione. In memoria di Paola, Milano 1975, s. 359 (commento ai vv. 4-5 dell'Ep CVIII 23); Y.M. Duval, Traces de lecture du "Peri Archôn” d'Origene avant le départ de Rome de Jérôme en 385. L'Ep. 39, la mort des enfants et la préexistence des âmes, in: Jérôme entre l'Occident et l'Orient. Études Augustiniennes, 
Inoltre, specie nel IV secolo, la diffusione del monachesimo accrebbe nella mentalità e nel sentire del popolo di Dio una sorta di „classificazione dei gradi della santità", secondo una certa interpretazione dei testi paolini, favorevole al primato della vita continente e tollerante come un minor male le nozze. Anche nella maggioranza dei Padri emerge l'idea dell'inferiorità del matrimonio rispetto alla verginità, in quanto considerato come conseguenza del peccato originale e non la condizione uscita dalle mani del Creatore ${ }^{3}$.

Nella Grande Chiesa, il deprezzamento del matrimonio dovuto all'ipertrofia dell'ideale ascetico arrivò così quasi a comprometterlo nella sua fondamentale bontà. Il problema, infatti, si spostò al dato della sessualità umana ridotta, secondo i canoni di tale mentalità, a realtà infralapsaria non appartenente alla verità antropologica dell'immagine di Dio. Un'ulteriore teoria di ,angelismo" dei progenitori nel paradiso terrestre, anche riguardo alla ipotetica procreazione asessuata, sganciò poi la teologia del matrimonio dal suo fondamentale riferimento biblico. Il disprezzo per la condizione umana, la storia, la materia ed il corpo influirono pure sull'interpretazione della novità apportata da Cristo. Alla motivazione escatologica del consiglio evangelico della continenza volontaria si aggiunse presto quella protologica estranea al contesto neotestamentario. La questione sulla verginità e sul matrimonio passò, pertanto, dalla funzione alla natura, con tutte le conseguenze che questo significò per l'ambito dogmatico, morale ed etico.

Benché il „no" al matrimonio fosse rimasto nel contesto dei movimenti ereticali ed extraecclesiali, tuttavia non restò senza influenza sulla tradizione patristica e contribuì notevolmente a sviluppare quell'atteggiamento di riserva nei riguardi delle nozze, che porterà i più a ritenerle un ,rimedio alla concupiscenza”, una „concessione” alla debolezza ed una ,necessità" per la procreazione. Infine, si può affermare che la rottura dell'equilibrio tra verginità e matrimonio, causata dalla mortificazione di quest'ultimo e dalla esaltazione della prima, non fu che il punto d'arrivo logicamente conseguente al rapporto compromesso tra carne e spirito o tra dimensione terrena e quella celeste ${ }^{4}$.

par Y.M. Duval, Paris 1988, 139-151; H. Crouzel, Virginité et Mariage selon Origène, Paris-Bruges 1961.

${ }^{3}$ Cf. G.D. Gordini (a cura di), Il „popolo di Dio” nel IV secolo. Gli „stati religiosi”, in: A. Fliche - V. Martin, Storia della Chiesa, III/1, Torino 1972, 413-419; T. Camelot, Les traités „De virginitate” au IV siècle, „Etudes Carmélitaines” 31(1952) t. 2: Mystique et continence, s. 173192.

${ }^{4}$ Cf. R. Cantalamessa, Etica sessuale e matrimonio nel Cristianesimo delle origini. Bilancio di una ricerca, in: Etica sessuale e matrimonio nel Cristianesimo delle origini, Milano 1976, 423-451; T. Špidlík, La spiritualità dell'Oriente cristiano, 192-193; E. Testa, Il Peccato di Adamo nella Patristica (Gen III), 13-15.119-156; C. Pietri, Il matrimonio cristiano a Roma (IV-V secolo), in: Storia vissuta del popolo cristiano, Torino 1985, 114-121; P. Brown, Il corpo e la società, Torino 1992, 334-353. 
In linea con il pensiero dominante su tutta questa complessa problematica, Girolamo applicherà il significato della parabola del seminatore di Mt 13, 3-23 al rapporto verginità, vedovanza e matrimonio, esplicitando così quella „scala di santità" attribuita ai diversi stati di vita:

\begin{abstract}
„Il grano da semina, buttato su una terra buona, produce il cento, il sessanta e il trenta per uno; così dicono i Vangeli. Il primo posto ce l'ha il cento in quanto raffigura la corona della verginità; il secondo ce l'ha il sessanta, che è simbolo del patire delle vedove; e che il trenta esprima il contratto matrimoniale ce ne danno conferma le stesse dita che si congiungono" ${ }^{5}$.
\end{abstract}

La spiegazione fornita dal Dottore dalmata è tratta dalle interpretazioni dei numeri in uso nell'antichità con una tecnica rigorosamente codificata sull'alfabeto delle dita ${ }^{6}$. Da questo schema, comunque, le nozze non escono ,sconfitte". Infatti, esse trovano nel Cristianesimo un primo riconoscimento di cui Girolamo stesso si fa araldo, sia per autodifesa, sia per consapevolezza di nuovi valori che saranno poi lentamente acquisiti come tali integralmente ${ }^{7}$.

L'introduzione ad opera della Rivelazione d'una dimensione nuova della persona e del suo destino segnò, sì, la valorizzazione di realtà che, se nell'ottica terreno-materialistica della vita erano svilite, in quella orientata ai beni del Regno dei cieli acquistavano più degna considerazione. L'insegnamento del santo di Betlemme indica l'assestamento progressivo dei nuovi valori nella recezione del suo tempo. Al martirio si sostituisce la verginità e, dopo di essa, la relativizzazione degli altri stati di vita si ordina offrendo pure al matrimonio un posto nella scala assiologica. Doveva considerarsi già tale posizione una conquista culturale rispetto al Cristianesimo primitivo o al paganesimo.

${ }^{5}$ Epistula (= Ep) 123,8, CSEL 56, 82; cf. Ep 22, 15, CSEL 54, 163: „centesimus et sexagesimus fructus de uno sunt semine castitatis”; - Ep 49, 3, CSEL 54, 354: „,centesimum et sexagesimum et tricesimum fructum de una terra et de una exposuimus semente generari, licet multum in numero differat".

${ }^{6}$ Cf. Ep 49, 2, CSEL 54, 353-354; Adversus Jovinianum (= Adv Jovin) I 3, PL 23, 223-224: „Triginta referuntur ad nuptias. Nam et ipsa digitorum conjunctio quasi molli se complexans osculo, et foederans, maritum pingit et conjugem. Sexaginta vero ad viduas, eo quod in angustia et tribulatione sunt positae. Unde et superiori digito deprimuntur; quantoque major est difficultas expertae quondam voluptatis illecebris abstinere tanto majus est praemium. Porro centesimus numerus [...] de sinistra trasfertur ad dexteram, et iisdem quidem digitis, sed non eum manu, quibus in laeva nuptae significantur et viduae, circulum faciens, exprimit virginitatis coronam"; H.I. Marrou, Storia dell'educazione nell'antichità, Roma 1950, 213-214.

${ }^{7}$ Cf. Ep 49,3, CSEL 54, 354:,,et quisquam tam iniquus lector erit, ut non ex meis dictis, sed ex suo me sensu iudicet? et certe multo clementiores erga coniugia fuimus omnibus paene Latinis et Graecis tractatoribus, qui centesimum numerum ad martyres referunt, sexagesimum ad uirgines, tricesimum ad uiduas, atque ita fit iuxta illorum sententiam, ut de bona terra et de patris familiae semine excludantur mariti”; P.F. Beatrice, Martirio ed ascesi nel sermone pseudo-ciprianeo „De centesima, sexagesima, tricesima”, in: Paradoxos Politeia. Studi patristici in onore di Giuseppe Lazzati, Milano 1979, 3-24. 
Fu la conseguenza di questa ad aprire, quindi, quel vivace dibattito alla ricerca dei valori in esso presenti, al quale ancora non si era in grado di dare una risposta né adeguata, né esauriente. Girolamo pertanto si trovò nell'imbarazzo teologico di una fede che muoveva i primi passi verso l'esigenza di una coscienza nuova la quale, in nome di Cristo, faceva crollare vecchi pregiudizi acquisiti con l'istanza d'una profondissima conversione morale e di un'altrettanto ampia e conseguente rivoluzione etica ${ }^{8}$.

\section{VERGINITÀ E MATRIMONIO}

Per leggere correttamente i testi di Girolamo sul raffronto verginità e matrimonio è necessario ricordare che egli ha voluto essere l'apostolo della continenza. I suoi scritti, sollecitati da più parti, escono dalla sua diretta esperienza in base alla quale il grande Dottore ha offerto i propri consigli sui problemi essenziali della vita spirituale. Si può affermare che, al di là di indicazioni pratiche, egli creò piuttosto uno spirito divenuto uno dei fermenti più attivi, attraverso i secoli, nel promuovere una concezione evangelica dell'esistenza, dell'abbandono del mondo, dell'austerità della vita cristiana e della condizione dello stato religioso. Talvolta, la crudezza di certe sue espressioni in difesa della realtà soprannaturale e dell'amore a Cristo ha delle punte sconcertanti. Pur lasciando grande libertà, e nel rispetto della discrezione personale, lo Stridonense trasmette le idee di un'epoca di varie e forti esperienze ascetiche, nella quale la rinuncia al matrimonio è considerata l'atto più significativo dell'autentico amore evangelico' ${ }^{9}$.

Il pensiero geronimiano riflette, dunque, la sua naturale austerità, radicalità e rigorosità, ed esprime sempre un amore proteso ad esaltare ogni sacrificio $^{10}$. La notevole differenza che anche al di fuori delle aspre polemiche l'asceta dalmata pone tra i due stati di vita è dettata dallo schema Legge e Vangelo: cronologicamente il matrimonio è la prima legge dell'Antico Testamento, la verginità l'ultima nell'eccellenza del Nuovo ${ }^{11}$.

${ }^{8}$ Cf. F. Valli, Un eretico del IV secolo. Gioviniano, „Didaskaleion” 3(1924) 1-66; G. Jouassard, La personalité d'Helvidius, in: Mélanges J. Saunier, III, Lyon 1944, 139-154; A.M. Triacca, "Verginità cristiana” e „matrimonio cristiano”, in: Il celibato per il Regno, Milano 1977, 77-129.

${ }^{9}$ Cf. F. Cavallera, Saint Jérôme et la vie parfaite, „Revue d'Ascetique et de Mystique” 2(1921) 101-127; P. Petitmengin, Saint Jérôme et Tertullien, in: Jérôme entre l'Occident et l'Orient, 43-59; H. Hagendahl, Latin Fathers and the Classics, Göteborg 1958, 142-182.

${ }^{10}$ Cf. Ep 49, 5, CSEL 54, 357: ,in Ecclesia diximus dona diuersa et aliud donum uirginitatis, aliud nuptiarum $[\ldots]$ sed inter donum et donum magna diuersitas est”.

11 Cf. Adversus Helvidium (= Adv Helv) 20, PL 23, 213: „Et quia de comparatione virginitatis et nuptiarum sum aliqua dicturus, obsecro lecturos ne me putent nuptiis detraxisse in virginum laude, et aliquam fecisse distantiam inter sanctos veteris Testamenti et novi, id est, inter eos qui habuere conjugia, et hos qui a complexu mulierum penitus recesserunt, verum pro conditione 
Questa serena premessa trova subito dopo un'analisi tendenziosa a favore della verginità, svolta sui testi paolini della prima lettera ai Corinti, e cade infine nel luogo comune delle ,angustiae nuptiarum”. Essa trova, però, anche l'affermazione della distinzione di „qualità” tra i due doni in questione: „Ho chiamato oro la verginità ed il matrimonio argento" 12 .

Tale subordinazione delle nozze viene estesamente ,giustificata” con convinzione da Girolamo nella sua opera polemica contro il monaco Gioviniano. Quest'ultimo sosteneva che le vergini, le vedove e le maritate, se battezzate, avevano equivalenti meriti e le loro opere il medesimo valore, non ponendo così differenza alcuna tra i vari stati di vita. Inoltre, egli affermava l'essere ugualmente i battezzati invincibili dal demonio, il digiunare od il mangiare la medesima cosa, se fatta con riconoscenza a Dio, e la ricompensa dal cielo uguale per tutti coloro che avessero vissuto con fiducia la fedeltà al proprio battesimo.

Ogni sforzo di perfezione veniva così scoraggiato nell'appiattimento degli ideali evangelici, già messi in pericolo da una cultura dominante di prassi pagana e da un Cristianesimo di massa adagiatosi dopo l'epoca dei martiri. Di questo Girolamo è preoccupato quando si getta nella polemica in corso, che già aveva condannato come eretico Gioviniano. I suoi toni, tuttavia, eccedono sul versante opposto, suscitando non meno scalpore nell'opinione pubblica ${ }^{13}$.

Il pensiero più cauto di Girolamo è che vergini e coniugati siano tenuti ciascuno alle opere della fede secondo il proprio stato. In ciò, la chiamata alla santità è equivalente, tanto che egli biasima quei monaci o chierici i quali, pur praticando la continenza, denigrano tuttavia lo stato prescelto. Il monaco di Betlemme invoca la coerenza nella testimonianza, da parte di chi vi si è impegnato, di valori considerati superiori ed illuminanti la „novitas” del Vangelo.

E' tra una condizione e l'altra che si pone l'ordine diverso delle perfezioni perseguibili: il matrimonio è connesso alla morte, la verginità, invece, alla vita eterna $^{14}$. Per tale motivo la dimensione delle nozze va superata con un perfe-

temporum alii eos tunc subjacuisse sententiae, et alii nos, in quos fines saeculorum decurrerunt"; Ep 107, 13, CSEL 55, 304: ,primam dei sententiam secunda euangelii lege mutares”; Adv Jovin I 29, PL 23, 263: „Qui servivimus in lege nuptiis, virginitati in Evangelio serviamus”.

${ }^{12}$ Ep 49, 3, CSEL 54, 354: , aurum uirginitatem, argentum diximus matrimonium”; ibid., 14, CSEL 54, 373; Adv Jovin I 40, PL 23, 280-282.

13 Cf. Ep 49, 2, CSEL 54, 351: „Reprehendunt in me quidam, quod in libris, quos aduersum Iouinianum scripsi, nimius fuerim uel in laude uirginum uel in suggillatione nuptarum"; ibid., 20, CSEL 54, 385: „Grande piaculum, euersae sunt ecclesiae, orbis audire non potest, si mundiorem uirginitatem diximus esse quam nuptias".

${ }^{14}$ Cf. Adv Jovin I 37, PL 23, 273: „Puto quod et nuptiarum finis mors sit. Fructus autem sanctificationis, qui vel ad virginitatem, vel ad continentiam pertinet, vita pensatur aeterna"; $E p$ 22, 18, CSEL 54, 167: ,et ad extremum morte morieris' finis iste coniugii”; $E p$ 49, 2, 6, 14, CSEL 54, 352-353, 358-359, 371-375; Ep 50, 3, CSEL 54, 390: ,ne uirgo uel continens [...] maritos uirginibus conparet"; - H.C.V. Eijk Ton, Marriage and Virginity, Death and Immortality, in: Epektasis. Mélanges patristiques offerts au Cardinal Jean Daniélou, Paris 1972, 209-235. 
zionamento concepito nella perfetta castità: si tratta di passare, in progressione qualitativa, dall'ordine della creazione compromessa dal peccato all'ordine della redenzione proiettata al possesso dei beni futuri. Emblematico è quanto, fuori da ogni tensione polemica, l'apostolo della continenza scrive alla figlia spirituale Eustochio circa la condizione della sorella Blesilla rimasta vedova:

„Tua sorella Blesilla, maggiore di te in età, ma inferiore per ideale ascetico, è rimasta vedova sette mesi dopo lo sposalizio [...]. Ella ha perduto, a un tempo, la corona della verginità e le gioie del matrimonio. D'accordo, occupa ancora il secondo grado della castità. Puoi però immaginare che spina dev'essere per lei, in certi momenti, vedere ogni giorno nella sorella quel bene che ella ha perduto e sapere che la sua castità, tanto più difficile a conservarsi dopo aver provato il piacere, otterrà una ricompensa minore" ${ }^{15}$.

Dal brano si intende che la subordinazione del matrimonio alla verginità è causata dall'inferiorità di ideale ascetico nel contenuto del primo rispetto a quello della seconda. A questo condizionamento della cultura cristiana del IV secolo, si aggiunge l'umano pregiudizio verso il piacere sessuale, di cui si gode lecitamente nelle nozze, e la considerazione per la verginità anche fisica. Secondo tale idea, la vedova è compianta perché perde il meglio delle gioie terrene ed il meglio della ricompensa futura. In compenso sale al secondo grado di castità e, qualora non si sposi, avrà premio eterno sì inferiore alla vergine, ma superiore alla coniugata. Il grado di santità è, dunque, vincolato allo stato di vita più che alla personale fedeltà alla vocazione abbracciata ${ }^{16}$.

Il „matrimonio che perdura nel suo stato" di coniugalità è di per sé penalizzato: solo la perfetta castità lo riscatta; ma allora, non sono esorcizzate le nozze in quanto tali? L'asceta dalmata si trincera dietro l'apostolo Paolo a giustificazione del proprio pensiero: se questi predica il celibato, dove sta l'errore delle sue idee? ${ }^{17} \mathrm{La}$ sua opinione guarda positivamente al superamento netto dei doveri coniugali in nome di un matrimonio ,purificato dal sesso”. Infatti, la prospettiva geronimiana ritiene la continenza quale l'elemento fon-

15 Ep 22, 15, CSEL 54, 162-163; cf. Ep 47, 2, CSEL 54, 345: „Desiderio e Serenella vivono in continenza nel matrimonio"; Ep 49, 7, CSEL 54, 359-361; ibid., 6, 10, CSEL 54, 359, 365: „si egeris patienter, coniux mutabitur in sororem [...] in secundo et tertio gradu uiduas ponimus et maritatas, et heretico furore damnare dicimus nuptias?".

${ }^{16}$ Cf. Ep 49, 11, CSEL 54, 366-367: ,ecclesia nuptias, sed nuptias in suo opere permanentes subicit, non damnat, nec abicit, sed dispensat. In potestate uestra est, si uelitis, secundum pudicitiae gradum scandere; quid indignamini, si in tertio stantes nolitis ad superiora properare?"; Ep 22, 2 , CSEL 54, 146: „, habet enim et maritatae ordinem suum, honorabiles nuptias et cubile immaculatum".

${ }^{17}$ Cf. Ep 48, 3, CSEL 54, 348-349; Ep 49, 15, CSEL 54, 375-376: „Ex quibus uniuersis perspicuum est me nihil noui de uirginibus nuptisque dixisse, sed maiorum in omnibus secutum esse sententiam"; Cf. anche: Ep 22, 21, CSEL 54, 173-174 (Girolamo commenta brani di 1Cor 7, in particolare i vv. 32-34). 
damentale della santità anche coniugale, perché l'essere un solo spirito è la condizione della Nuova Alleanza che, nella logica della Risurrezione supera l'Antica dell'essere una sola carne ${ }^{18}$.

Nella Lettera ad Eustochio il monaco di Betlemme, comunque, dichiara di non volere né esaltare la verginità, né mettere in luce „, i fastidi” delle nozze. Tuttavia, elenca gli aspetti negativi delle seconde e riguardo alla prima non nasconde la propria simpatia. Egli stesso se ne accorge e cerca di prevenire probabili contestatori ${ }^{19}$.

Benché affermi di non intendere un confronto fra un bene ed un male, il vero pensiero di Girolamo differenzia matrimonio e verginità proprio su un canone simile, distinguendo sottilmente il ,non peccare” del primo dal „bene facere" della seconda, poi abilmente corretto col rapporto tra il bene ed il meglio. E' nell'opera polemica contro Gioviniano, appunto, che egli mette a nudo le proprie idee più convinte in materia:

„Tra nozze e verginità corre la medesima differenza che passa tra il non peccare ed il fare il bene; o meglio, per usare un'espressione meno forte, tra il bene e il meglio" 20 .

Quando nella Lettera a Pammachio cercherà di spiegare tale sua presa di posizione, l'austero fautore della vita ascetica si rivelerà, forse inconsciamente, ancor più radicale nei suoi giudizi, paragonando il „,meglio" della verginità al bene del matrimonio ed il "meglio" di quest'ultimo al peccato della fornica$z^{21 o n e}{ }^{21}$. L'insegnamento geronimiano, dunque, diviene ancor più pericoloso perché, commentando 1 Cor 7, 3, scivola decisamente arrivando a dire che le nozze sono un minor male.

${ }^{18}$ Cf. La coppia nei Padri, 75-89; D. Dumm, The Theological Basis of Virginity according St. Jerome, Latrobe Pa. 1961, 25-51.

${ }^{19}$ Cf. Ep 22, 2, CSEL 54, 146: ,molestias nuptiarum”; ibid., 19, CSEL 54, 168: „Dicat aliquis: et audes nuptiis detrahere, quae a domino benedictae sunt?' non est detrahere nuptiis, cum illis uirginitas antefertur. Nemo malum bono conparat; glorientur et nuptae, cum a uirginibus sunt secundae”; Ep 49, 11, CSEL 54, 366: „nam cum proposuissem, quod dominus isset ad nuptias in Cana Galilaeae, [...] addidi: 'qui enim semel iuit ad nuptias, semel docuit esse nubendum' [...] in domo magna non solum uasa aurea et argentea, sed et lignea et fictilia, et alia esse in honorem, alia in contumeliam, et quicumque se mundauerit, eum futurum esse uas honorabile et necessarium, in omne opus bonum praeparatum"; Adv Jovin I 40, PL 23, 281-282.

${ }^{20}$ Adv Jovin I 13, PL 23, 243: „Tantum est igitur inter nuptias et virginitatem, quantum inter non peccare, et bene facere; inmo, ut levius dicam, quantum inter bonum et melius".

${ }^{21}$ Cf. Ep 49, 7, CSEL 54, 380-381: ,,si dixero: 'melius est uirginem esse quam nuptam', bono melius praetuli; si autem alterum gradum fecero: 'melius est nubere quam fornicari', ibi non bono melius, sed malo bonum praetuli, et multa diuersitas est inter id melius, quod nuptiis, et inter id, quod fornicationi anteponitur”; Adv Jovin I 9, PL 23, 233: ,Si per se nuptiae sunt bonae, noli illas incendio comparare: sed hic simpliciter, bonum est nubere. Suspecta est mihi bonitas ejus rei, quam magnitudo alterius mali malum esse cogit inferius. Ego autem non levius malum, sed simplex per se bonum volo". 
Non ricorre mai in Girolamo l'argomento che, abbassare la dignità del matrimonio è per conseguenza indebolire l'eccellenza della verginità, anzi pare il contrario: l'esaltare la verginità offre in qualche modo la possibilità di rivalutare anche le nozze. Infatti, questa conclusione non è quella accettata come ultima da Girolamo, il quale mostra chiaramente quanto il giudizio sul matrimonio sia relativo al termine di paragone con cui lo si intende raffrontare. Se, come san Paolo, si cita il fuoco travolgente della concupiscenza, la valutazione dello stato coniugale ne esce in termini negativi. Se, invece, si rapporta alla realtà dello stato verginale, il contenuto che gli si attribuisce è positivo. L'asceta sembra propendere per quest'ultima soluzione, benché non si possa non notare l'assenza dottrinale di un pensiero riguardante il matrimonio per se stesso. L'argomento più proposto a sfavore dello stato coniugale rispetto alla vita continente è quello delle ,angustiae nuptiarum”, le quali mortificano la preghiera e rivelano quanto le nozze siano un „legame”. Innanzi tutto, la tensione conflittuale è posta da Girolamo tra tempo e finalità dell'unione coniugale e tempo per la preghiera a Dio. Ne deriva che, conseguito l'unico scopo legittimo del matrimonio, la procreazione, la coppia debba restituire il primato alla preghiera ${ }^{22}$.

Dei „fastidi” del matrimonio Girolamo parla anche ad Eustochio, alla quale l'abile maestro spirituale scrive di non aver intenzione di elencarli, benché di fatto poi li elenchi: ,il seno che ingrossa, il bimbo che vagisce, le rivali che fanno dannare, le faccende domestiche che non lasciano un attimo di quiete e tutti quei beni che si credono sorgente di felicità, ma che pensa la morte a portar via" 23 .

La conclusione geronimiana sulle ,angustiae nuptiarum” è che alla fine, i frutti di tanta tribolazione, appartenendo alla realtà peritura, sono destinati tutti all'annientamento della morte. La felicità che quei beni sembrano offrire è, quindi, apparente e vacua, vanità effimera iscritta nel tempo transeunte.

${ }^{22}$ Cf. Ad Eph III, 5, 25, PL 26, 566: „Liberorum ergo [...] in matrimonia opera concessa sunt [...] Hoc legens omnis vir, et uxor intelligant, sibi post conceptum, magis orationi quam connubio serviendum. Et quod in animalibus et bestiis ipso naturae jure praescriptum est, ut praegnantes ad partum usque non coeant, hoc in hominibus sciant arbitrio derelictum, ut merces esset ex abstinentia voluptatum".

${ }^{23}$ Ep 22, 2, CSEL 54, 146: ,nec enumeraturum molestias nuptiarum, quomodo uterus intumescat, infans uagiat, cruciet paelex, domus cura sollicitet et omnia, quae putantur bona, mors extrema praecidat”; ibid., 2, 22, CSEL 54, 146, 174: „sed ut intellegeres tibi exeunti de Sodoma timendum esse Loth uxoris exemplum $($ Gen 19,26) ... Quantas molestias habeant nuptiae et quot sollicitudinibus uinciantur, in eo libro, quem aduersus Heluidium de beatae Mariae perpetua uirginitate edidimus, puto breuiter expressum”; Ep 49, 18, CSEL 54, 381, 382: „Illud uero ferre quis possit, quod in me reprehenditur, quare exponens capitulum apostoli, in quo de coniugibus scripsit: tribulationem carnis habebunt huiusmodi' (1Cor 7,28) ... quae hic condemnatio matrimonii est, si infantum uagitus, filiorum mortes, uxorum abortia, damna domus et cetera istius modi tribulationem diximus nuptiarum?". 
Concisa e puntuale la frase dell'eremita di Betlemme si presenta emotivamente carica di attese deludenti e di intensa amarezza, specie per l'ideale d'una giovane. Tuttavia, è con un linguaggio ancor più crudo per le immagini usate, che l'austero dalmata parla del matrimonio alla vedova Furia, la quale ne ha conosciuto, quindi, la condizione. Si tratta forse di una delle pagine più sconcertanti contro lo stato coniugale, che la letteratura patristica conservi, tanta è l'efficacia figurativa in negativo che lo descrive:

„Quante spine porti con sé il matrimonio, l'hai constatato a tue spese durante la vita matrimoniale. Te ne sei saziata fino alla nausea, come gli Ebrei della carne di quaglia. Il tuo palato ha provato l'amarezza infinita del fiele, hai vomitato cibi acidi e malsani, hai mitigato l'arsura dello stomaco: perché vorresti ancora ingerire cose che ti sono state di danno? Proprio come un cane che ritorna ai cibi vomitati o un maiale al fango dove s'è avvoltolato?"24.

La soverchia pagina termina col problema della discendenza, la quale non sempre è data e, comunque, qualora lo fosse, sarebbe proprio come desiderata? ${ }^{25}$

Ma l',,angustia” peggiore dei coniugati nell'adempimento del loro stato è, per il monaco dalmata, l'incompatibilità di questo con una vita di preghiera. Costituirà per Girolamo proprio tale motivo il grande argomento spirituale invocato contro il matrimonio nella convinzione che a ciò precisamente si riferisse l'apostolo Paolo in 1Cor 7, 33-34:

„Di là garriscono i bambini, schiamazza la servitù, i figli pendono dagli occhi e dalla bocca, si devono fare i conti, disporre le spese; da una parte la schiera ben armata dei cuochi pesta le carni, dall'altra mormorano insieme la folla delle tessitrici. Intanto viene annunziato che è arrivato il marito con gli amici [...] Rispondimi, di grazia, fra tutte queste cose, dov'è il pensiero di Dio?"26.

${ }^{24}$ Ep 54, 4, CSEL 54, 468-469: „Quid angustiarum habeant nuptiae, didicisti in ipsis nuptiis et quasi coturnicum carnibus usque ad nauseam saturata es. Amarissimam choleram tuae sensere fauces, egessisti acescentes et morbidos cibos, releuasti aestuantem stomachum: quid uis rursus ingerere, quod tibi noxium fuit? canis reuertens ad uomitum et sus ad uolutabrum luti"'(2Pt 2, 22). Bruta quoque animalia et uagae aues in easdem pedicas retiaque non incidunt"; Adv Helv 18, PL 23, 212: ,naturae contumelias, novem mensibus uterum insolescentem, fastidia, partum, sanguinem, pannos".

${ }^{25}$ Cf. Ep 54, 4, CSEL 54, 469: „omnes habent filios, quae habuere matrimonia, et, quibus nati sunt liberi, suo generi responderunt!"; L. Mirri, Il monachesimo femminile secondo sant'Ambrogio, Vicenza 1991, 48-53.

${ }^{26}$ Adv Helv 20, PL 23, 214; ibid., 20, PL 23, 214: ,tamen ipsa dispensatio domus, liberorum educatio, necessitates mariti, correctio servulorum, quam a Dei cogitatione non avocent?"; Ep 22, 22, CSEL 54, 174: „,cum apostolus sine intermissione orare nos iubeat et, qui in coniugio debitum soluit, orare non possit, aut oramus semper et uirgines sumus, aut orare desinimus, ut coniugio seruiamus". 
Il quadretto di movimentata vita domestica che Girolamo dipinge è pure efficace nel rendere la contrapposizione tra la vita nel mondo e quella nella solitudine del silenzio monastico, favorevole al raccoglimento ed all'unione con Dio. Riportando poi l'esempio biblico di Abramo e Sara, il santo Dottore espone l'intera logica del suo pensiero riguardo alla donna. Questa nel matrimonio si troverebbe a vivere le angosce della maledizione divina a seguito del peccato originale, dalla quale la menopausa la libera. Inoltre, vi è l'impossibilità per entrambi gli sposi di assolvere all'ordine dell'Apostolo di pregare incessantemente, a meno che non rinuncino a vivere la sessualità ${ }^{27}$.

Nell'insegnamento geronimiano colpisce l'idea che la donna cessi di essere tale per il solo fatto di entrare in menopausa. Anzi, questo evento della sua natura la libererebbe dalla "maledizione di Dio" connessa concretamente con le ,angustiae nuptiarum” che l'asceta non ricusa di presentare alle figlie spirituali in toni assai duri. Tuttavia, bisogna aggiungere un'altra rivelazione: la donna in menopausa è ancora sposata, ma non esercitando più il potere generativo, è considerata idonea alla preghiera e guida al marito. Lo statuto primitivo di Gen 3, 16 per la donna viene inoltre annullato con un capovolgimento della situazione: non più la donna soggetta al dominio del marito, ma l'uomo obbediente alla sposa come il Signore ingiunse ad Abramo: „Ascolta la parola di Sara in quanto ti dice, ascolta la sua voce (Gen 21, 12)".

Se alla donna pone davanti le fatiche dei molteplici compiti in cui cimentarsi come sposa, madre e padrona di casa, così all'uomo Girolamo presenta il matrimonio come un laccio dal quale, una volta legati, si deve restare vincolati. L'opposto, ovviamente, è la libertà, secondo l'ermeneutica di 1Cor 7, 27 che egli ricorda ai propri interlocutori. Tra questi c'è il soldato romano Esuperanzio, al quale, in proposito, con non poca finezza psicologica, ricordandogli la norma data dall'Apostolo, egli appunto scrive , di non cercare un legame che è l'opposto della libertà”, poiché „,chi è sottomesso ai doveri coniugali è legato e chi è legato è schiavo, ma chi invece è sciolto, è libero"28. Per un uomo, poi, il matrimonio si rivela una schiavitù duplice se si considera che, oltre quella della carne, comporta pure quella dell'intelligenza. Infatti, esso, nell'idea geronimia-

27 Cf. Adv Helv 20, PL 23, 214: „Quae non est in partus anxietatibus et dolore, quae deficientibus menstrui cruoris officiis, mulier esse dessit, a Dei maledictione fit libera: nec est ad virum conversio ejus, sed e contrario vir subjicitur ei [...] et sic incipiunt vacare orationi. Quia quandiu in conjugio debitum solvitur, orandi praeteritur instantia"; Adv Jovin 7, PL 23, 230-231; E. Prinzivalli, Donna e generazione nei Padri della Chiesa, in: La donna nel pensiero cristiano antico, a cura di U. Mattioli, Genova 1992, 79-94.

${ }^{28}$ Ep 145, CSEL 56, 306: „illud autem prudentiae tuae breuiter significasse sufficiat, ut memineris apostolicae sententiae: uinctus es, inquit, uxori? ne quaeras solutionem; solutus es? ne quaeras uxorem' (1Cor 7, 27), id est alligationem, quae solutioni contraria est. qui igitur seruit officio coniugali, uinctus est; qui uinctus est, seruus est; qui autem solutus est, liber est"; Ep 49, 67, CSEL 54, 358-361; ibid., 21, CSEL 54, 387: „in nostro arbitrio est uel Lazarum sequi uel diuitem". 
na, preclude la possibilità della vita teoretica, perché „,non si può dare attenzione parimenti alla moglie ed alla filosofia" ${ }^{29}$.

Per questa concezione Girolamo dipende esplicitamente dalla tradizione classica, nella quale i filosofi pagani, pur riconoscendo legittimità al matrimonio, ne discutevano la convenienza. Il problema non era posto tanto in termini morali, quanto piuttosto pratici, in attinenza alla carriera del sapiente greco ed al rispetto dell',otium” romano. Teofrasto, Aristotele, Plutarco, Crisippo, Epicuro, Seneca e Cicerone, il quale evitò di risposarsi per non avere impedimenti alla vita speculativa, sono sull'argomento le fonti del santo Dottore dalmata. Egli non solo ne utilizza i passi o li cita come autorità in materia, ma ne sponsorizza pienamente il pensiero, condividendone le varie posizioni concordi nella risposta negativa circa la conciliabilità tra stato coniugale e dedizione allo studio ${ }^{30}$.

L'ideale della perfezione cristiana realizzata rompendo i rapporti con il mondo tramite una rinuncia totale, è tale in Girolamo da condurlo ad un'esaltazione della verginità, esclusiva nei riguardi del matrimonio. Egli lo testimonia con un passo che non lascia dubbi riguardo alla sua convinzione che soltanto sulla prima si innesta il „,novum” assoluto dei doni di Dio:

„'Ecco, la Vergine concepirà e partorirà un figlio' (Is 7, 14). Se la verginità non fosse preferita alle nozze, perché lo Spirito Santo non ha scelto una donna maritata o una vedova? Infatti, tempo addietro, visse Anna, figlia di Fanuel, della tribù di Aser, rinomata per la sua pudicizia e sempre dedita alla preghiera e al digiuno nel tempio di Dio. Se la preghiera, le buone opere e i digiuni, da soli senza la verginità, possono meritare la venuta a noi dello Spirito Santo, una di queste donne avrebbe potuto pure essere la madre del Signore" ${ }^{31}$.

Quanto alle nozze, presentandosi inoltre di ostacolo per la vita ascetica, devono essere necessariamente superate al fine di conseguire una santità possibile, a quanto pare, unicamente nella via della continenza ${ }^{32}$. La gravissima dichiarazione diviene accettabile solamente se letta in chiave di castità coniugale, intesa come santità del matrimonio cristiano. Questa è esercitata

${ }^{29}$ Adv Jovin I 48, PL 23, 291: „,non posse se uxori et philosophiae pariter operam dare”.

${ }^{30}$ Cf. Adv Jovin I 47, 49, PL 23, 289,293: „Fertur aureolus Theophrasti liber de Nuptiis', in quo quaerit an vir sapiens ducat uxorem [...]. Non est ergo uxor ducenda sapienti [...] enim impediri studia philosophiae: nec posse quemquam libris et uxori et noster Seneca de matrimonio libros, ex quibus et superiora nonnulla sunt, et ista quae subjicimus: Amor formae, rationis oblivio est, et insaniae proximus; foedum minimeque conveniens animo sospiti vitium"; ibid., 48, PL 23, 291-293; C. Tibiletti, Verginità e matrimonio in antichi scrittori cristiani, Napoli 1969, 27-55.

${ }^{31}$ Adv Jovin I 32, PL 23, 266: E.A. Clark, Jerome: The Exaltation of Christian Virginity, in: E.A. Clark - H. Richardson, Women and Religion, New York-London 1977, 53-68.

${ }^{32}$ Cf. Adv Helv 21, PL 23, 214: „Non negamus viduas, non negamus maritatas, sanctas mulieres inveniri; sed quae uxores esse desierint, quae in ipsa necessitate conjugii virginum imitentur castitatem". 
nella generosa accoglienza dei figli, delle prove della vita e di una grazia di castità vissuta quale dono dello Spirito e non obbligata dall'età avanzata.

Comunque, l'unica lode geronimiana allo stato coniugale in se stesso si riferisce al medesimo come a mera realtà strumentale nel fine particolare al quale è ordinata e per il quale è legittimato: la procreazione e, in essa, la nascita di futuri nuovi consacrati nel celibato per il Regno dei cieli ${ }^{33}$. Le rose, l'oro e la perla contrapposte alle spine, alla terra ed alla conchiglia, negli esempi che egli porta, esprimono figurativamente ed inequivocabilmente il pensiero dello Stridonense su verginità e matrimonio: „la prima, qualità destinata a servizi più nobili; la seconda, a usi più umili" ${ }^{34}$.

\section{VEDOVANZA E SECONDE NOZZE}

Nel valutare il rapporto tra la continenza e il matrimonio, ancor più esplicativo delle idee di Girolamo è quanto egli scrive alle vedove o afferma sulle seconde nozze. Innanzi tutto c'è da considerare che nel IV secolo il movimento monastico, di cui l'asceta dalmata divenne araldo in modo particolarmente forte, iniziò ad assumere la condizione della vedovanza al pari di quella della verginità consacrata ${ }^{35}$. Questo spirito propugnatore della continenza liberò così lo stato vedovile dal divenire nella Chiesa soltanto una categoria di assistite, spiritualmente ed economicamente.

E' in particolare il monachesimo geronimiano a rendere testimonianza, invece, della duplice attività ecclesiale delle vedove cristiane. In primo luogo, in campo economico, furono le più facoltose tra loro che, con il proprio patrimonio ereditato e messo operosamente a frutto, sostennero la nascente vita cenobitica $^{36}$. Vi è interessante notare la solidarietà materiale femminile che

${ }^{33}$ Cf. Ep 22, 20, CSEL 54, 170: „Laudo nuptias, laudo coniugium, sed quia mihi uirgines generant: lego de spinis rosas, de terra aurum, de conca margaritum [...] plus honorantur nuptiae, quando, quod de illis nascitur, plus amatur”; Adv Jovin I 27, PL 23, 260: „Nam si salvatur mulier in filiorum generatione, et liberorum numerus salus matrum est, cur addidit, $\mathrm{Si}$ permanserint filii in charitate et sanctificatione cum castitate' (1Tm 3,15)? Tunc ergo salvabitur mulier, si illo genuerit filios, qui virgines permansuri sunt".

${ }^{34}$ Ep 49, 2, CSEL 54, 353: ,ita nuptias recipimus, ut uirginitatem, quae de nuptiis nascitur, praeferamus”; ibid., 11, CSEL 54, 366: „alia esse in honorem, alia in contumeliam”.

35 G.D. Gordini, Forme di vita ascetica a Roma nel IV secolo, „Scrinium Theologicum” 1(1953) 38-45; D. Dumm, The Theological Basis of Virginity, 133-141; A. Nazzaro, Figure di donne cristiane: la vedova, in: Atti del II Convegno nazionale di studi su „La donna nel mondo antico”, Torino 18-20 aprile 1988, 197-219; G. Ramis, La consagración de la mujer en las liturgias occidentales, Roma 1990, 193, 204-206.

${ }^{36}$ Cf. Ep 52, 5, CSEL 54, 423: „multas anus nutrit ecclesia, quae et officium praebeant et beneficium accipiant ministrando"; Ep 54, 13, CSEL 54, 479: „sanctarum uirginum et uiduarum societatem adpete”; ibid., 14, CSEL 54, 481: „Habes opes, facile tibi est indigentibus uictus subsidia ministrare; quod luxuriae parabatur, uirtus insumat; nulla nuptias contemptura timeat egestatem. 
non prevedeva l'inserimento della donna nel mondo del lavoro e nella società. Per coloro che si sottraevano alla patria potestà, il matrimonio era l'unica fonte di sostentamento. Il Cristianesimo, col movimento ascetico, portò anche in questo campo la prima rivoluzione sociale di promozione della donna, con la proposta ideale e concreta di un'esistenza autosufficiente.

L'organizzazione della vita comune come mezzo sicuro non solo spiritualmente, ma anche economicamente all'adempimento della promessa di continenza, trovò una certa risposta positiva nel monachesimo femminile, tanto che Girolamo dà testimonianza di una vedova romana la quale, addirittura, era circondata da un centinaio di vergini consacrate! $!^{37}$ Le vedove giovani, e che avessero desiderato vivere in continenza, erano comunque sollecitate a lavorare per procurarsi in qualche modo il necessario al proprio sostentamento senza pesare sulla collettività ecclesiale. Ciò fa pensare ad un numero elevato di donne che scegliessero tale stato monastico.

Il santo Dottore interpreta tra l'altro le parole di san Paolo in 1Cor 7, 29 in chiave di autonomia economica e non affettiva, collegandole al passo di $1 \mathrm{Tm} 5$, 3: le vedove veramente tali, cioè prive di figli e di congiunti, sono le non autosufficienti materialmente e l'età dei 60 anni starebbe per lo più ad indicare quelle non più in grado comunque di esserlo con un lavoro proprio ${ }^{38}$. Di per sé lo stato di vedovanza consacrata non comportava particolari doveri se non quello di una vita spirituale più intensa e di una condotta più austera, pur nell'adempimento delle normali pratiche come la cura dei figli, dei poveri e degli elementari bisogni quotidiani ${ }^{39}$.

Redime uirgines, quas in cubiculum saluatoris inducas, suscipe uiduas"; $E p$ 79, 5, CSEL 55, 93: "quae uidua non huius auxilio sustentata est?".

37 Cf. Ep 123, 1, 13, CSEL 56, 73, 89: „Benigna mater quartum et decimum inplens uiduitatis annum centenario uirginum choro cingitur [...] quantae uirgines et uiduae absque ulla sorde rumoris suam substantiolam gubernarunt?”; Ep 127, 3, CSEL 56, 147, 148: „nostra uidua [...] semper in comitatu suo uirgines ac uiduas".

${ }^{38}$ Cf. Ep 123, 5, CSEL 56, 78: „si ergo apostolus pauperes uiduas - eas tamen, quae adulescentulae sunt et nulla debilitate franguntur - cogit suis manibus laborare, ne grauetur ecclesia et possit anus uiduas sustentare, qua excusatione utitur, quae opibus mundi affluit, quae potest etiam aliis ministrare?”; ibid., 5, CSEL 56, 77, 78: „nec sibi in eo annorum puellarium debet uidua blandiri, quod non minus sexagenariae electionem praecipit [...] sed de his uiduis disputat, quae suorum nutriuntur alimentis, quae filiorum et nepotum ceruicibus inponuntur; quibus imperat, ut discant domum suam colere et remunerari parentes et sufficienter eis tribuere, ut non grauetur ecclesia et possit certis uiduis ministrare, de quibus scriptum est: honora uiduas, quae uere uiduae sunt' (1Tm 5,3), hoc est, quae omni suorum auxilio destitutae, quae manibus suis laborare non possunt, quas paupertas debilitat aetasque conficit, quibus deus spes est et omne opus oratio. Ex quo intellegi datur adulescentulas uiduas exceptis his, quas excusat infirmitas, uel suo labori uel liberorum ac propinquorum ministerio deligari".

${ }^{39}$ Cf. Ep 123, 3, 13, CSEL 56, 74-75, 87-89; Ep 127, 3, CSEL 56, 147-148; Ep 79, 8-9, CSEL 55, 97-99. 
Anche per quanto concerne l'aspetto spirituale, non si può dire che le vedove costituissero una categoria di sole assistite nell'istituto ecclesiale. Infatti, esse assunsero parte attiva nella vita ascetica sia con l'esempio della loro vita, sia con l'organizzazione di veri e propri circoli culturali, nei quali si tenevano frequenti incontri di spiritualità. Nella vita comune o in queste fraternità simpatizzanti dell'ideale monastico, le vedove fungevano talvolta da autentiche „madri spirituali”, o per iniziativa spontanea propria, o per sollecitazione di maestri e direttori spirituali come lo stesso Girolamo. I Cenacoli dell'Aventino ne sono l'esempio più fulgido e da essi deriverà la fondazione dei monasteri geronimiani a Betlemme, dei quali costituirono come una preistoria istituzionale ${ }^{40}$.

Al riguardo, spicca la testimonianza della vedova Lea, nel panegirico della quale il grande Dalmata dichiara che „si era data al Signore in modo così totalitario da essere messa a capo del monastero e da diventare madre di vergini" ${ }^{41}$. Il monastero di cui si parla è uno di quei circoli spirituali che promossero e diffusero il movimento ascetico femminile nell'Urbe. Lea, come Marcella e Paola, era il perno di queste riunioni di vergini e di vedove nella propria casa, che ne fu la sede. A queste nobili donne veniva dato il titolo di „monacha” o di ,, ancilla Christi”, a seconda dell'intenzione di voler sottolineare la loro encomiabile vita ascetica od il particolare „status” di unione con Dio che liberamente avevano scelto dopo la morte del coniuge ${ }^{42}$.

Le condizioni per essere annoverate tra le vedove cristiane erano quelle già indicate da san Paolo nella lettera a Timoteo: provate qualità morali e, soprattutto, l'essere stata la sposa di un solo marito. Riguardo ad entrambi i requisiti, Girolamo è satirico ed intransigente ogni qual volta ha occasione di parlarne, come quando scrive alla giovane Geruchia per esortarla a restare nella vedovanza $^{43}$. Interessante è il parallelismo con le disposizioni ecclesiastiche concernenti l'accesso al sacerdozio per uomini sposati.

${ }^{40}$ Cf. Ep 79, 9, CSEL 55, 97, 98: , habetum tecum uiduarum et uirginum choros [...] semper in manibus tuis diuina sit lectio et tam crebrae orationes, ut omnes cogitationum sagittae, quibus adulescentia percuti solet, huiusce modi clipeo repellantur".

${ }^{41}$ Cf. Ep 23, 2, CSEL 54, 212: ,equidem conuersationem Leae nostrae quis possit digno eleuare praecomio? Ita eam totam ad dominum fuisse conuersam, ut monasterii princeps, mater uirginum fieret".

42 Cf. Ep 39, 5, CSEL 54, 304: „si Christianam et monacham Christianam”; Ep 108, 6, CSEL 55, 311: „nesciebat matrem, ut Christi probaret ancillam”.

43 Cf. Ep 123, 5, CSEL 56, 78-79: ,simulque considera, quod uidua non eligatur nisi unius uiri uxor [...] non solum enim ab officio sacerdotali digamus excluditur, sed et ab elemosyna ecclesiae, dum indigna putatur stipe, quae ad secunda coniugia deuoluta est”; Ep 22, 16, CSEL 54, 164: ,fuge, quas uiduas necessitas fecit, non quo mortem optare debuerint maritorum, sed quo datam occasionem pudicitiae libenter arripere. Nunc uero tantum ueste mutata pristina non mutatur ambitio [...] et, quia maritorum expertae dominatum uiduitatis praeferunt libertatem, castae uocantur et nonnae et post cenam dubiam apostolos somniant"; Adv Jovin I 14, PL 23, 243-244. 
Nessuno speciale atto pubblico e liturgico contrassegnava la consacrazione nella vita ascetica di una vedova che intendesse „renuntiare saeculo" ed ,aeternae pudicitiae dedicare" 44 . Il proposito di vivere in continenza era generalmente manifestato col mutamento di abito: vesti semplici e dimesse sostituivano gli abiti lussuosi e ricercati ad indicare il distacco col mondo ${ }^{45}$. Inoltre, come le vergini, anche le vedove pare si velassero ${ }^{46}$.

Girolamo conclude la sua opinione sull'autentica vedova, di cui descrive nell'epistola a Furia portamento e ,toelette”, con le parole di Paolo in 1Tm 5, 6, dove si afferma che „una vedova che vive nei piaceri è una morta che vive" ${ }^{, 47}$. Tutte le lettere geronimiane a vedove contengono un'esortazione alla vita continente ed un'esaltazione di essa inversamente proporzionale al disprezzo per le seconde nozze ${ }^{48}$.

Oltre che al movimento monastico che premeva da un lato, il problema di queste ultime interessava dall'altro la stessa dottrina del matrimonio circa l'indissolubilità del coniugio e la monogamia. L'argomento che porta l'asceta dalmata è coerente con le motivazioni protologiche, ontologiche ed escatologiche nelle quali si inquadra il suo pensiero sulla verginità e sull'origine od il significato delle nozze. Così egli, infatti, si esprime in attinenza alle prime:

„La creazione dei primi uomini ci insegni a rifiutare ogni matrimonio successivo al primo. Adamo è una persona singola, e altrettanto unica è Eva, anzi, è una costa di lui che viene staccata per farne una donna. Ma poi, ciò che era stato diviso viene riunito grazie al matrimonio" 49 .

Ritorna qui il motivo geronimiano dell',,unum" come perfezione ed integrità della creatura, per il quale aveva persino ricondotto, con filologia forzata,

${ }^{44}$ Cf. Ep 39, 5, CSEL 54, 304: „cum sciam toto renuntiasse te mundo”; Ep 127, 2, CSEL 56, 146: „si uellem nubere et non aeternae me cuperem pudicitiae dedicare”.

${ }^{45}$ Cf. Ep 39, 1, CSEL 54, 294: ,humilitas uestium non, ut in plerisque, tumentes animos arguebat, sed cum interiori se mente deiecerat, inter ancillarum uirginum cultum dominamque nihil medium, nisi quod in eo facilius dinoscebatur”; ibid., 3, CSEL 54, 300: „quod habitu a matronis ceteris separato tibi quasi religiosior uidebaris?”; Ep 38, 3, CSEL 54, 290-291: ,at scandalizat quempiam uestis fuscior: scandalizet"; $E p$ 54, 7, CSEL 54, 473; Ep 79, 1, CSEL 55, 87-88; ibid., 7, CSEL 55, 96: „tu uero, quae in tumulo mariti sepelisti omnes pariter uoluptates, quae litam purpurisso et cerussa faciem super feretrum eius lacrimis diluisti, quae pullam tunicam nigrosque calceolos candidae uestis et aurati socci depositione sumpsisti, nihil habes necesse aliud nisi perseuerare”; Ep 127, 4, CSEL 56, 148: ,uilique opertus palliolo pugnat contra tineas uestium sericarum".

${ }^{46}$ Cf. Ep 38, 4, CSEL 54, 291: „tunc crines ancillulae disponebant et mitellis crispantibus uertex artabatur innoxius; nunc neglectum caput scit sibi tantum sufficere, quod uelatur".

${ }^{47}$ Cf. Ep 54, 8, CSEL 54, 473-474: „,ceterum uidua, 'quae in deliciis est' (1Tm 5, 6), - non est meum, sed apostoli - 'uiuens mortua est'; quid sibi uult hoc, quod ait: 'uiuens mortua est'? uiuere quidem uidetur ignorantibus et non esse peccato mortua, sed Christo [...] mortua est”.

${ }^{48}$ Cf. Ep 123, 1, CSEL 56, 72: „saepe ad uiduas scripsimus”.

${ }^{49}$ Ep 123, 11, CSEL 56, 84. 
„virgo” a „vir”. In questo brano, Girolamo restituisce al matrimonio come istituzione la sua bontà intrinseca, in quanto diverrebbe salvaguardia dell'unità nella distinzione sessuale della coppia umana.

L'Adamo viene presentato come ,la persona singola", cioè l'uno, all'interno del quale, e non dall'esterno, si colloca la differenziazione del femminile. Tale significato è attribuito ad Eva, ,altrettanto unica” in quanto costola di Adamo che, grazie al matrimonio, viene ricollocata al suo posto nell'uno. Il richiamo alla costola sottolinea efficacemente l'elemento di caratteristica diversa appartenente all'insieme dell'uno e non una natura estranea ad esso. In riferimento a ciò il biblista di Betlemme poi specifica che ,'per questo l'uomo lascerà padre e madre e si unirà a sua moglie' (Gen 2, 24); e cioè, per precisione: non alle sue mogli'",50.

Passando quindi all'ermeneutica neotestamentaria, Girolamo si rifa' alla teologia paolina del „sacramentum magnum” di Ef 5, 32, applicata sia al simbolismo dell'unione tra Adamo ed Eva che a quello del vincolo CristoChiesa $^{51}$. L'unità del matrimonio per il santo dalmata è inscindibile dalla sua unicità nel tempo e nell'eternità, essendo entrambi i requisiti il mistero teologico della natura delle nozze in sé nel ,,sacramentum magnum” di Adamo ed Eva e di Cristo e della Chiesa. Anzi, il pensiero viene da lui ampliato toccando anche il problema delle eresie come di realtà ibride ed extraecclesiali:

„L'unica Eva è la madre di tutti i viventi e l'unica Chiesa è la madre di tutti $i$ cristiani. Come quel maledetto di Lamech ha spezzato la monogamia prendendo due mogli $(\mathrm{Gen} 4,19)$, allo stesso modo gli eretici lacerano la Chiesa in più chiese; ma queste, a quanto dice l'apostolo Giovanni, si devono chiamare sinagoghe del diavolo più che comunità di Cristo $(\mathrm{Ap} 2,9)^{, 52}$.

E con questa contrapposizione tra Antico e Nuovo Testamento, Girolamo espone senza mezzi termini quale sia la sua opinione riguardo alle seconde nozze, verso le quali non mancherà di usare espressioni durissime ed esempi paradossali $^{53}$. A difesa della propria causa su questo argomento, il monaco di

${ }^{50}$ Cf. Ep 123, 11, CSEL 56, 84: ,'propter quod relinquet homo patrem et matrem et adhaerebit uxori suae' (Gen 2, 24), certe non uxoribus”; Ad Eph III 5, 32, PL 26, 569: ,quod reliquerit Dominus noster atque Salvator Patrem suum Deum, et matrem suam coelestem Jerusalem, et venerit ad terras propter suum corpus Ecclesiam, et de suo eam latere fabricatus sit (Gv 19,34)".

51 Cf. Ep 123, 11, CSEL 56, 84: „quod testimonium Paulus edisserens ad Christum refert et ad ecclesiam, ut et primus Adam in carne et secundus in spiritu monogamus sit"; Ep 49, 9, CSEL 54, 363-364: „Christum in carne uirginem, in spiritu monogamum, quod unam haberet ecclesiam, noster in reliquis sermo testatus est"; Ad Eph III 5, 22-23, PL 26, 564.

52 Ep 123, 11, CSEL 56, 84; cf. Adv Jovin I 16, PL 23, 246.

53 Il caso limite di un vedovo risposatosi 20 volte e di una vedova 22 volte, sposatisi a loro volta tra di loro dopo essersi incontrati al cimitero a piangere sulle tombe dei relativi coniugi, è narrato da Girolamo con forte sarcasmo nell' $E p$ 123, 9, CSEL 56, 82-83; ibid., 12, CSEL 56, 85: "quod si et nobis iure conceditur, adhinniamus ad omnes feminas et in exemplum Sodomae et 
Betlemme si appella persino all'autorità dell'esempio dei pagani nelle usanze religiose dell'età classica e, più ancora, a quelle di eroiche donne barbare che preferirono la morte alla violenza ${ }^{54}$.

Nella foga tipica del suo temperamento, il Dalmata si lascia sfuggire una condanna non solo alle seconde nozze, ma al matrimonio in sé quando conclude la propria argomentazione affermando che sposarsi un'altra volta per una vedova è agire ,una seconda volta contro il pensiero di Dio". Il ritratto impietoso sulle seconde nozze, comunque, emerge dall'esasperazione descrittiva che egli impiega per dimostrarne l'inferiorità rispetto alle prime, emulando spesso contenuti e stile del De Monogamia di Tertulliano ${ }^{55}$.

Addirittura scrivendo alla vedova Salvina ${ }^{56}$ ed alla vedova Furia ${ }^{57}$, lo Stridonense impiega espressioni che toccano la nostra sensibilità come una ferita a quei sentimenti ed a quell'amore dei quali il matrimonio è anzitutto costituito. Tuttavia, nell'asceta di Betlemme rappresentano una convinzione spontanea in ordine alla scala di santità prefigurata sui diversi gradi di castità nel rapporto nozze-continenza e pure un freno pagato al clima prevaricante dei tempi. E' precisamente da questa scala assiologica che egli esclude assolutamente le seconde nozze ${ }^{58}$.

Girolamo è consapevole, tuttavia, di non poter sostenere su base scritturistica, e da essa dogmatica, l'illiceità delle seconde nozze, ammesse dall'apostolo Paolo e, quindi, entrate nella tradizione della Grande Chiesa. Allora è la sua interpretazione a porsi in senso restrittivo riguardo ad un problema, sul quale

Gomorrae ab ultima die deprehendamur uendentes et ementes, nubentes et nuptum tradentes, ut tunc sit finis coniugii, quando terminus uitae"; Ep 49, 18, CSEL 54, 383: , habeat quaelibet octauum maritum, et esse se uni deneget prostitutam".

${ }^{54}$ Cf. Ep 123, 8, CSEL 56, 81: „Quod igitur barbarae castitati non potuit inferre captiuitas, hoc matrona nobilis faciet et experietur alterum uirum, quae priorem aut bonum perdidit aut malum experta est, ut rursum contra iudicium dei facere nitatur?"; ibid., 7, CSEL 56, 80-81.

${ }_{55}$ Cf. Ep 123, 5, CSEL 56, 76: ,uiuente uiro mulier alligata est et mortuo soluta. ergo matrimonium uinculum est et uiduitas solutio"; C. Micaelli, L'influsso di Tertulliano su Girolamo: le Opere sul matrimonio e le seconde nozze, ,Augustinianum” 19(1979) 415-429.

56 Cf. Ep 79, 7, CSEL 55, 96: ,audiant haec mulieres, quae sollicitae sunt, quomodo placeant uiris. Comedant carnes, quae carnibus seruiunt, quarum femor despumat in coitum, quae ligatae maritis generationi ac liberis dant operam. quarum uteri portant fetus, earum et intestina carnibus inpleantur"; D.S. Wiesen, St. Jerome as a Satirist, New York 1964, 140: così commenta questa lettera a Salvina: „We can conjecture how well Princess Salvina received this arrogant missive from Jerome's failure even to mention her again".

${ }^{57}$ Cf. Ep 54, 6, CSEL 54, 472: ,quin potius doleat [pater tuus], quod et uirginitatem frustra amiseris et fructus perdideris nuptiarum [...] arripe, quaeso, occasionem et fac de necessitate uirtutem"; Ep 22, 15, CSEL 54, 162-163.

58 Cf. Ep 123, 8, CSEL 56, 82: ,digamia in quo erit numero? immo extra numerum [...] ut in eo se putet esse laudabilem, si scortis melior sit, si publicarum libidinum uictimas superet, si uni sit prostituta, non pluribus”; Adv Jovin I 14, PL 23, 244: „Melius est enim licet alterum et tertium, unum virum nosse, quam plurimos: id est, tolerabilius est uni homini prostitutam esse, quam multis". 
non riesce ad attenuare per nulla la propria esasperata austerità di asceta ${ }^{59}$. Soltanto la pressione delle sette eretiche, come i Montanisti, mette in imbarazzo l'intransigenza geronimiana in materia, tanto che a Marcella, la quale chiedeva spiegazioni in proposito, il Dottore dalmata espone i medesimi concetti, ma con diversa sfumatura ${ }^{60}$.

Se l'idea della „tolleranza” è più positiva che altrove, Girolamo però non ne esce né convinto né convincente. Spicca infatti, in primo luogo, il contrasto tra ciò che „,non desideriamo”, opinione tra l'altro personale estesa di riflesso al plurale della collettività ecclesiale, e l',ordine" dell'Apostolo. In secondo luogo, non giudica in realtà egli pure „scellerato” un secondo matrimonio, anzi, ,un meretricio o poco più su"? Non è lo stesso Autore che chiama digami o trigami o lussuriosi quanti, vedovi o vedove, si risposano? E' sempre colui che scrive a Geruchia che se una vedova prenderà un quarto o un quinto marito nulla la distinguerà dalle meretrici e che, dunque, ,deve badare, sotto ogni punto di vista, a non oltrepassare i primi limiti della castità”, poiché, „se va al di là ed infrange quel pudore che conviene alle matrone, si avvoltolerà sfrenatamente in ogni sorta di lussuria" ${ }^{\circ 1}$.

Certamente Girolamo ha dinanzi gli abusi della corrotta società romana, ma quando poi deve trattare di un caso particolare di convivenza si mostra alquanto indulgente e prudente nel giudizio, lasciando pieno spazio alla comprensione umana, alla pietà ed alla misericordia. Lo fa nell'elogio funebre di Fabiola, indirizzato all'amico Oceano. Il problema delicato del dramma di questa donna, separatasi giovanissima da un marito depravato e poi unitasi ad un altro uomo, trova nel cuore del santo asceta quello spazio ampio e sereno

${ }^{59}$ Cf. Ep 123, 3, CSEL 56, 75: „uult apostolus alterum matrimonium praeferens digamiam fornicationi, secundum indulgentiam dumtaxat, non secundum imperium"; Ep 42, 8, CSEL 54, 361363; riguardo a 1Cor 7, 6: Adv Jovin I 8, 37, PL 23, 231, 274, 275: „'Hoc autem, inquit, dico juxta indulgentiam, non juxta imperium'. Et mussitamus adhuc nuptias non vocare indulgentiam, sed praeceptum, quasi non eo modo et secunda et tertia matrimonia concedantur, quasi non et fornicatoribus per poenitentiam fores aperiantur Ecclesiae, quodque his est majus, et incestis? [...] Concedit quidem Deus nuptias, concedit digamiam, et, si necesse fuerit, fornicationi et adulterio praefert etiam trigamiam [...] Aliud est voluntas Dei, aliud indulgentia".

${ }^{60}$ Cf. Ep 41, 3, CSEL 54, 313: ,nos secundas nuptias non tam adpetimus, quam concedimus Paulo iubente, ut uiduae adulescentulae nubant; illi in tantum scelerata putant iterata coniugia, ut, quicumque hoc fecerit, adulter habeatur".

${ }^{61}$ Cf. Ep 123, 8, CSEL 56, 81: ,si statim secundum perdiderit, sortietur et tertium? et si ille dormierit, in quartum quintumque procedet, ut quo a meretricibus differat? Omni ratione uiduae prouidendum est, ne castitatis primos excedat limites. quos si excesserit et uerecundiam ruperit matronalem, in omnem debacchabitur luxuriam"; Ep 49, 18, CSEL 54, 382: ,porro, ubi de digamis et trigamis diserentes diximus: melius est, licet alterum et tertium, unum uirum nosse quam plurimos', id est: tolerabilius est uni homini prostitutam esse quam multis [...] ego etiam nunc libera uoce proclamo non damnari in ecclesia digamiam, immo nec trigamiam, et licere quinto et sexto et ultra, quomodo et secundo marito, nubere"; Ep 54, 15, CSEL 54, 482-483; Adv Jovin I 7 , PL 23, 229-230. 
col quale avrebbe forse potuto accettare la condizione di una vedova risposatasi. Ci troviamo di fronte alla trattazione di un caso di separazione o di divorzio:

„Lei, dunque, si è separata, come mi dicono, da un vizioso [...]. Se la colpa che le si fa è quella di essere andata con un altro uomo dopo aver ripudiato suo marito, non ho difficoltà ad ammettere che abbia sbagliato, ammesso però che non si possa dimostrare che lì si verificava un caso di necessità" ${ }^{\prime 2}$.

Cosa intende Girolamo con l'ultima clausola? Cosa significa in questo contesto ,un caso di necessità” di contrasto aggiunto al pensiero precedente e come eventuale assoluzione del suo contenuto? Ci troviamo qui davanti all'ipotesi di un caso di annullamento? Sta di fatto che subito dopo egli si appella all'autorità di Paolo e tratta del caso come di quello di una vedova, con molta benevolenza ${ }^{63}$.

Benché non vi sia difesa dei sentimenti di questa creatura in simile esperienza, vi è almeno della sua sincerità, che ha bandito l'ipocrisia d'un falso matrimonio esponendosi alla vergogna di un'illecita convivenza. Ad essa, tra l'altro, rimase fedele fino alla morte, dopo aver vissuto pure un'autentica vedovanza a seguito della perdita di quello che possiamo considerare il vero affetto di Fabiola. Il tono dell'apostolo della continenza, comunque, in questo elogio funebre, non è più accondiscendente di quando, costretto ad ammettere le seconde nozze per le persone vedove, afferma che ,se questi matrimoni non vengono condannati, è anche vero che non vengono lodati”, in quanto ,sono di sollievo ad una debolezza, non di onore alla continenza" ${ }^{\text {64 }}$.

La tolleranza, pertanto, verso la liceità di essi è motivata dalla debolezza della fragilità umana, stesso motivo di fondo per il quale è ammesso da Girolamo il matrimonio in sé. Egli vorrebbe, piuttosto, che quella ,debolezza” fosse vinta e non ,avvallata” nell'istituto delle nozze. Non può vedere così alcun bene in sé nel matrimonio, eccetto che quel primordiale ritorno all'uno di Adamo ed Eva, figura di Cristo e della Chiesa, e della generazione... di vergini, cioè di creature per l'ordine della Redenzione in proiezione escatologica di avvento del Regno di Dio.

${ }^{62}$ Ep 77, 3, CSEL 55, 39: „dimisit ergo, ut aiunt, uitiosum [...] sin autem arguitur, quare repudiato marito non innupta permanserit, facile culpam fatebor, dum tamen referam necessitatem".

${ }^{63}$ Cf. Ep 77, 3, CSEL 55, 39: „'melius est, inquit apostolus, nubere quam uri' (1Cor 7, 9). Adulescentula erat, uiduitatem suam seruare non poterat. Videbat aliam legem in membris suis repugnantem legi mentis suae et se uinctam atque captiuam ad coitum trahi. Melius arbitrata est aperte confiteri inbecillitatem suam et umbram quandam miserabilis subire coniugii quam sub gloria uniuirae exercere meretricium".

${ }^{64}$ Cf. Ep 49, 18, CSEL 54, 383: „sed quomodo non damnantur hae nuptiae, ita nec praedicantur; solacio miseriae sunt, non laudi continentiae". 
Di questi unici due beni, appena ammessi dall'austero asceta di Betlemme, il primo è infranto dalle seconde nozze, alle quali quindi non appartiene; il secondo viene invece addirittura escluso in quanto „,chi è coerede può desiderare un erede per mezzo degli uomini?" 65 . Ma la posizione moderata del Dottore dalmata, perno di tutte le sue argomentazioni, potrebbe riassumersi in quanto scrive alla stessa Geruchia:

„Ma allora? Condannerei per caso le seconde nozze? Nient'affatto; mi limito a elogiare le prime. Radio forse i digami dalla Chiesa? Non sia mai detto; però quelli che hanno contratto le nozze una prima volta cerco di convincerli alla continenza" $" 66$.

\title{
WSTRZEMIĘŹLIWOŚĆ I MAŁŻEŃSTWO W NAUCE ŚW. HIERONIMA
}

\author{
(Sommario)
}

Artykuł bogaty w najnowszą literaturę przedmiotu składa się z dwóch zasadniczych części: w pierwszej przedstawiony jest stosunek św. Hieronima do małżeństwa i dziewictwa, które Strydończyk zgodnie z ówczesną tradycją wychwala i stawia wyżej od pierwszego (to jedynie dopuszcza ze względu na prokreację i pomnażanie liczby dziewic), w drugiej zaś omówiony jest rzadko poruszany przez współczesnych badaczy status wczesnochrześcijańskich wdów i ich działalności, oraz problem niechętnie widzianego w starożytności chrześcijańskiej powtórnego małżeństwa.

${ }^{65}$ Cf. Adv Jovin I 48, PL 23, 291: „Haeredem nimirum desiderabit hominem coheres Christi?"; ibid., I 47, PL 23, 289-290; Ep 54, 4, 15, CSEL 54, 469, 481-483.

${ }^{66}$ Ep 123, 8, CSEL 56, 81: „damnamus secunda matrimonia? minime, sed prima laudamus. Abicimus de ecclesia digamos? absit, sed monogamos ad continentiam prouocamus"; Ep 49, 6, CSEL 54, 358: „Aperiant, quaeso, aures obtrectatores mei et uideant me secundas et tertias nuptias in domino concessisse; qui secundas et tertias non damnari, primum potui damnare matrimonium?". 